\title{
Primary Cutaneous Gamma Delta T Cell Lymphoma: A Clinicopathological Analysis
}

\author{
Li Tang1, Yu Li ${ }^{*}$ \\ ${ }^{1}$ Department of Pathophysiology, Chongqing Medical University, Chongqing, China \\ ${ }^{2}$ Department of Pathology, Institute of Neuroscience, Chongqing Medical University, Chongqing, China \\ Email: tangli@cqmu.edu.cn, *liyu@cqmu.edu.cn
}

How to cite this paper: Tang, L. and Li, Y. (2019) Primary Cutaneous Gamma Delta T Cell Lymphoma: A Clinicopathological Analysis. Journal of Biosciences and Medicines, 7, 22-26.

https://doi.org/10.4236/jbm.2019.75005

Received: February 26, 2019

Accepted: May 14, 2019

Published: May 17, 2019

\begin{abstract}
Primary cutaneous gamma-delta T-cell lymphoma (PCGD-TCL) is a very uncommon and extremely aggressive tumor, and is described in the newly revised World health organization for research and treatment of cancer classification of cutaneous lymphomas. A 43-year-old male patient presented with a 4 months history of cutaneous lesion over upper lip, without plaque and any constitutional symptom. Histopathological examination of skin biopsy revealed infiltration of atypical lymphocytes with hyperchromatic irregular nuclei. Immunophenotyping pattern of skin biopsy was compatible with PCGD-TLC. It is a highly aggressive tumor resistant to chemotherapy, immunotherapy, and radiation therapy. The GDTCL is characterized by a worse prognosis with a median survival of 15 months. Early diagnosis is essential and aggressive therapy is necessary.
\end{abstract}

\section{Keywords}

Cutaneous Gamma-Delta T-Cell Lymphoma, Hematoxylin and Eosin, Immunohistochemistry

\section{Introduction}

Cutaneous T-cell lymphoma (CTCL) is classified as a subtype of non-Hodgkin's lymphoma and is a heterogeneous group of T-cell malignancies. Primary cutaneous gamma-delta $(\gamma \delta$ ) T-cell lymphoma (PCGD-TCL) is a very uncommon and extremely aggressive tumor. It is recognized as a distinctive type of T-cell lymphoma composed of $\mathrm{T}$ cells that express the gamma-delta $\mathrm{T}$-cell receptor (TCR) in 2016 World Health Organization classification [1]. Only 40 cases of PCGD-TCL have been described in the literature. 


\section{Materials and Methods}

1) Patient A 43-year-old male patient presented with a 4 months history of cutaneous lesion. There was a single soybean size, non-tender and a bit hard nodule over upper lip without plaque and any constitutional symptom.

2) Methods The skin tissue was fixed with $4 \%$ PFA, dehydrated and embedded in paraffin. Two-micrometer frozen sections were prepared, and hematoxylin-eosin (HE) staining was performed to assess pathological changes. Expression of $\mathrm{CD} 2, \mathrm{CD} 3, \mathrm{CD} 5, \mathrm{CD} 7, \mathrm{CD} 8, \mathrm{CD} 4, \mathrm{CD} 20, \mathrm{CD} 21$ TIA-1, GrB, CD30, CD56, EBER, TCR $\gamma \delta$, TCR $\alpha \beta$ and Ki- 67 were analyzed by immunohistochemistry. Antigen visualisation was achieved by applying a standard streptavidin-perosidase (S-P) method.

\section{Results}

Hematoxylin and eosin stain of skin lesion revealed a normal epidermal morphology and structure, there were no atypical cells at the junction of epidermis and dermis. Dense infiltration of medium to large lymphoid cells was found in the dermis and subcutaneous tissues without epidermotropic lymphoid cell (Figure 1). Tumor cells infiltrate nerves involved in skin appendages and small blood vessels. Lacy structures were formed in the interstitial of fat cells. Fat necrosis could be seen, but there were no other inflammatory cells (especially without plasma cells). Immunohistochemistry showed positivity of $\mathrm{CD} 2, \mathrm{CD} 3$, $\mathrm{CD} 5$ and $\mathrm{CD} 7$, while $\mathrm{CD} 2$ and $\mathrm{CD} 5$ were decreased. Expression of CD8 was stronger than CD4. TIA-1, GrB and $\operatorname{TCR} \gamma \delta$ were also positive, and negative reactions for $\mathrm{CD} 30, \mathrm{CD} 56, \mathrm{EBER}, \mathrm{TCR} \alpha \beta, \mathrm{CD} 21$ and $\mathrm{CD} 20$; a small number (30\%) of cells was positive for Ki-67 (Figure 2). In sum, according to the WHO-EORTC classification, the results were consistent with $\gamma \delta$ T-cell lymphoma.

\section{Discussion}

Primary cutaneous gamma/delta T-cell lymphoma is a lymphoma composed of a clonal proliferation of mature, activated $\gamma / \delta$ T-cells with a cytotoxic phenotype. PCGD-TCL is extremely rare and represents approximately $1 \%$ of CTCL [2].
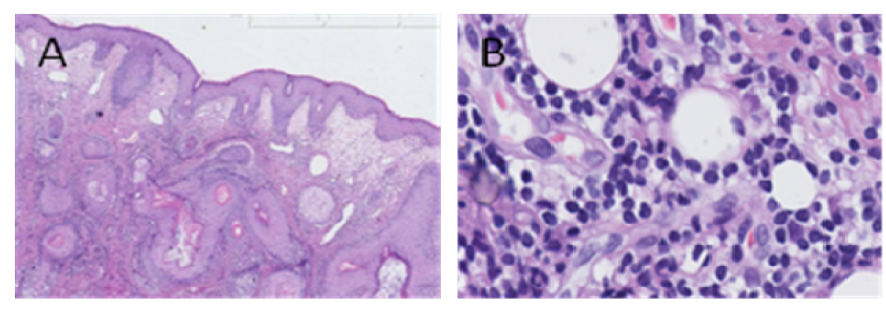

Figure 1. Histopathologic patterns and cytologic features of PCGD TCL. A, Epidermotropic pattern shows a dense lymphoid infiltrate predominantly into the epidermis $(\mathrm{HE} \times 10)$. B, Lymphoid infiltrate with a predominance of small lymphocytes with markedly irregular nuclear contours $(\mathrm{HE} \times 40)$. 

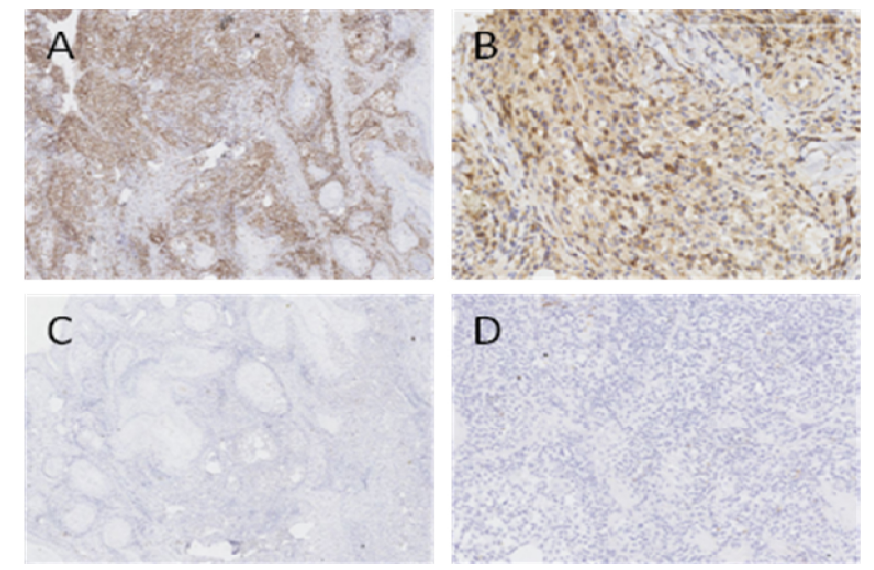

Figure 2. Immunophenotypic features of PCGD TCL. A, CD3 highlights a dense infiltrate of lymphocytes (IHC, $\times 10$ ); B, TCR $\gamma \delta$ highlights a dense infiltrate of lymphocytes (IHC, $\times 40$ ); C, CD30 is negative in the biopsy specimen (IHC, $\times 10$ ); D.EREB is negative $(\mathrm{IHC}, \times 10)$.

Chronic antigenic stimulation has been hypothesized to be involved in the pathogenesis of PCGD-TCL. The skin lesions are often generalized and involve the extremities. Most patients present with deep dermal or subcutaneous plaques or tumors, either with or without epidermal ulceration and necrosis. The involvement of mucosal and other extranodal sites is frequently noted, although lymph nodes, spleen, and bone marrow are rarely involved. There is no lymphatic tissue in skin originally, inflammatory stimulation or autoimmune disease cause the formation of lymphatic tissue, then develop to lymphoma. When diagnosing primary cutaneous lymphoma (within 6 months of initial diagnosis), there should be no lymphoma except the skin. The key differential diagnosis of cutaneous lymphoma is to distinguish from skin lymphoproliferative lesions and cutaneous pseudolymphoma. Once considered of primary cutaneous lymphoma, the invasive lymphoma should be excluded.

The distinct histological presentation of PCGD-TCL may be epidermotropic, dermal, and/or subcutaneous, and more than one of these three can be present in the same patient within the same biopsy specimen or in different biopsy specimens. Lichenoid or vascular interface dermatitis-like patterns of epidermal infiltration may occur, which may be associated with intraepidermal vesiculation and necrosis. Angiocentricity, angiodestruction, and tissue necrosis may be seen. In our case, there were a normal epidermal morphology and structure and no atypical cells at the junction of epidermis and dermis. Dense infiltration of medium to large lymphoid cells was found in the dermis and subcutaneous tissues without epidermotropic lymphoid cell. Lacy structures were formed in the interstitial of fat cells and fat necrosis could be seen.

Typically, the lymphoma cells are positive for CD2, CD3, CD56 and TCR $\gamma$ but are negative for $\mathrm{CD} 4, \mathrm{CD} 5, \mathrm{CD} 8$ and $\beta \mathrm{F} 1$ (TCR $\beta$ ), although a minor subset may be positive for $\mathrm{CD} 8$. The lymphoma cells also express cytotoxic proteins such as 
granzyme B and T-cell intracellular antigen 1 (TIA-1). Most cases harbor monoclonal rearrangements of the TCR $\gamma$ gene. When non-epidermophilic, dense $\mathrm{T}$ cell infiltration in dermis and subcutaneous tissue are presented, the positive rate of CD2, CD3, CD5, CD4, CD8 T cell markers, CD30, CD56, and whole B cell marker CD20 are needed to determine the density of $\mathrm{B}$ cells. $\mathrm{CD}^{+}$and $\mathrm{CD} 56^{+}$ $\mathrm{NK} / \mathrm{T}$ cell lymphoma, $\mathrm{CD} 4^{+} / \mathrm{CD}^{-}$, and $\mathrm{CD} 56^{-}$lymphoma got more aggressive clinical process. In our case, interesting immunophenotype were CD56 ${ }^{-}$CD5 decreased (not negative), $\mathrm{CD}^{+}$and $\mathrm{CD}^{+/-}$(not common $\mathrm{CD} 4^{-} / \mathrm{CD}^{-}$).

Primary cutaneous lymphoma is totally different with lymphoma of lymph nodes. Some lymphomas only occur in the skin, such as mycosis fungoides (MF). Some skin lymphomas are morphologically similar to lymph node lymphomas, but the immunophenotype, genetic and clinical characteristics are different, such as the follicular lymphoma. The immunophenotype and genetic characteristics of some skin lymphomas are similar with lymphoma in lymph node, but clinical features are different, such as primary skin follicular central cell lymphoma.

The differential diagnosises of a primary cutaneous gamma/delta T-cell lymphoma include MF (with $\gamma \delta \mathrm{T}$ cell phenotype), subcutaneous panniculitis-like T-cell lymphoma (SPTCL), primary anaplastic large cell lymphoma (cALCL), extranodal NK/T cell lymphoma, nasal type, primary cutaneous CD8-positive aggressive epidermotropic cytotoxic T-cell lymphoma.

PCGD-TCL is a highly aggressive tumor resistant to chemotherapy, immunotherapy [3], and radiation therapy. Most patients with PCGD-TCL have B-type symptoms and the outcome is usually poor [4] [5]. Early diagnosis is essential and aggressive therapy is necessary. The effectiveness of hematopoietic stem cell transplantation has been reported in some patients with PCGD-TCL [6]. Other treatment is challenging and will require further study.

\section{Conflicts of Interest}

The authors declare no conflicts of interest regarding the publication of this paper.

\section{References}

[1] Swerdlow, S.H., Campo, E., Pileri, S.A., et al. (2016) The 2016 Revision of the World Health Organization Classification of Lymphoid Neoplasms. Blood, 127, 2375-2390. https://doi.org/10.1182/blood-2016-01-643569

[2] Soon, C.W., Link, M., Kim, Y.H., et al. (2015) Primary Cutaneous Gamma-Delta T-Cell Lymphoproliferative Disorder in a 3-Year-Old Boy. Am J Dermatopathol., 37, 567-569. https://doi.org/10.1097/DAD.0000000000000185

[3] Koch, R., Jaffe, E.S., Mensing, C., Zeis, M., Schmitz, N. and Sander, C.A. (2009) Cutaneous Gamma/Delta T-Cell Lymphoma. JDtsch Dermatol Ges, 7, 1065-1067. https://doi.org/10.1111/j.1610-0387.2009.07209.x

[4] Toro, J.R., Beaty, M., Sorbara, L., et al. (2000) Gamma Delta T-Cell Lymphoma of the Skin: A Clinical, Microscopic, and Molecular Study. Arch Dermatol., 136, 
1024-1032. https://doi.org/10.1001/archderm.136.8.1024

[5] Rodriguez-Pinilla, S.M., Ortiz-Romero, P.L., Monsalvez, V., et al. (2013) TCRgamma Expression in Primary Cutaneous T-Cell Lymphomas. Am J Surg Pathol., 37, 375-384. https://doi.org/10.1097/PAS.0b013e318275d1a2

[6] Gibson, J.F., Alpdogan, O., Subtil, A., et al. (2015) Hematopoietic Stem Cell Transplantation for Primary Cutaneous $\gamma \delta$ T-Cell Lymphoma and Refractory Subcutaneous Panniculitis-Like T-Cell Lymphoma. J AM Acad Dermatol., 72, 1010-1015. https://doi.org/10.1016/j.jaad.2015.01.003 